Review

\title{
A Brief Review on WIMPs in 331 Electroweak Gauge Models
}

\author{
P.S. Rodrigues da Silva \\ Departamento de Fisica, Universidade Federal da Paralba, \\ Caixa Postal 5008, 58051-970, Jo ao Pessoa, PB, Brasil
}

\author{
Article history \\ Received: 01-01-2015 \\ Revised: $10-02-2015$ \\ Accepted: 29-05-2015 \\ E-mail: lemingue@gmail.com
}

\begin{abstract}
In this study we review the cold dark matter problem in the context of a class of models which are a simple extension of the electroweak standard model, where the gauge symmetry is dictated by the group structure, $\mathrm{SU}(3)_{\mathrm{c}} \otimes \mathrm{SU}(3) \mathrm{L} \otimes \mathrm{U}(1)_{\mathrm{X}}$. This model, in different versions, has been able to address many interesting facts not explained by the standard model. It would be just desirable that the dark matter issue could be contemplated as well, since the problem of the missing matter has become one of the greatest indications of incompleteness of currently established theories describing the known interactions and the cosmological evolution and content of the observed Universe. We do that by pointing out some of the successful steps in this direction and remarking some sources of difficulties concerning their phenomenological and theoretical implementation.
\end{abstract}

Keywords: Dark Matter, Electroweak Model, Cosmological Evolution

\section{Introduction}

The last decades have seen an unprecedented accumulation of astrophysical and cosmological evidence towards the missing matter paradigm, culminating in recent data from the Planck satellite (Ade et al., 2014) that have improved our knowledge of matter content of the Universe, revealing that a non-baryonic dark matter amounts to roughly $27 \%$ in contrast to $23 \%$ of WMAP data (Bennett et al., 2003), both in the context of the standard cosmological model with a cosmological constant, $\Lambda$. That lead us to fiercely conclude that there exists some new component (or components) in the energy content of the Universe which must correspond to some exotic particle in the framework of a particle physics model. If this is the right track and that is the view we adopt in this review, such a particle should present certain features to explain its stability, abundance, non-relativistic behavior at decoupling from thermal bath and evasiveness while offering the possibility of being detected soon (not mandatory but desirable). One of the most attractive possibilities is the so called Weakly Interacting Massive Particle (WIMP), a particle with mass in the few $\mathrm{GeV}$ scale (even a TeV) that interacts most like a neutrino does, thus gathering the right properties to fulfill these requirements to explain this unknown matter, usually called Cold Dark Matter (CDM).

Simple extensions to the Electroweak Standard Model (EWSM) can deal with this unsolved puzzle, like adding a singlet fermionic field, plus a symmetry to guarantee its stability (Pires et al., 2010a). Although simplicity may seem the appropriate path to attack any new riddle we may face, we look at such models more like a guide, hoping that something more fundamental and ambitious may be hiding under this cloak. This leads us to look for more intricate models available that might shed light on other questions not embraced by the EWSM, but can also deal with the CDM problem. They are many (see for instance (Jungman et al., 1996; Murayama, 2007; Dodelson and Widrow, 1994; Cheng et al., 2002; Martin, 2006) and references therein) and each one poses a new content or mechanism that opens up new phenomenological possibilities that can be tested in current, as the Large Hadron Collider (LHC) and future colliders. Here we focus on a specific class of models, which represents only a small increase in the electroweak gauge group compared to the EWSM, namely, the $\mathrm{SU}(3)_{\mathrm{c}} \otimes \mathrm{SU}(3)_{\mathrm{L}} \otimes \mathrm{U}(1)_{\mathrm{X}}$ gauge model, or 331 for short.

Some of the interesting features of these models are, among others, that anomaly cancellation and QCD asymptotic freedom demand that only three family of fermions should be present in the theory, explaining the family problem (Pisano and Pleitez, 1992); electric charge quantization is automatic in these models (Pires and Ravinez, 1998); the tiny neutrino mass can be naturally explained (Dias et al., 2005) in a version of the model where the right handed neutrino is in the same multiplet along with its partners that form the usual doublet under EWSM (Singer et al., 1980; Montero et al., 1993; Valle and Singer, 1983), or even considering effective operators in the minimal model where the third leptonic component is the right handed charged lepton 
(Pires et al., 2010b); the strong CP problem through Peccei-Quinn mechanism can be implemented, yielding a nonthermal candidate for the CDM problem, the axion (Dias et al., 2003); the Higgs physics probed by LHC can be easily accommodated and also allow for some room to new Physics phenomena (Caetano et al., 2013); the discrepancy between theory and experiment on $(\mathrm{g}-2)_{\mu}$ can be accounted for in some versions of the model (Pires and Ravinez, 1998; Ky et al., 2000). Besides, it is possible for some versions of 331 model to present a natural CDM candidate as a WIMP (Pires and Silva, 2007; Mizukoshi et al., 2011) and it is this specific topic in the context of 331 models that we want to discuss in this review.

This work is organized as follows: In section II we present the models which are the focus of this review; Next, in section III, we discuss the emergence of a WIMP in two specific versions of 331 models, basically arguing about the role of symmetries in protecting the WIMP from decaying and commenting about the results and additional complementary studies concerning these WIMPs in the context of these models; we then present some final remarks in section IV.

\section{The 331 Models with Neutral Fermions}

There are basically two kinds of 331 models which differ in the electric charge of the third component of the $\mathrm{SU}(3)_{\mathrm{L}}$ fundamental representation. One possibility is that this component possesses one unit of electric charge (Pisano and Pleitez, 1992) and the other is when it is electrically neutral (Singer et al., 1980; Montero et al., 1993; Valle and Singer, 1983). We are not considering the first possibility in this review as it does not seem to allow for any reasonable symmetry that the claimed CDM candidate is stable or metastable (Fregolente and Tonasse, 2003), although such an issue has been already addressed in (Dong et al., 2013), where the authors promote one of the triplet scalar fields to be inert by imposing a $Z_{2}$ symmetry. The other possibility, with a neutral fermion as the third component of the lepton triplet, splits into two alternatives: the first is to put the right handed partner of the EWSM neutrino, $\left(\mathrm{v}^{\mathrm{c}}\right)_{\mathrm{L}}$, as the third component of the leptonic triplet; the second is to introduce a new left handed neutral fermion, $\mathrm{N}_{\mathrm{L}}$, instead. The 331 model with a right handed partner for the EWSM active neutrino we call $331 \mathrm{RHv}$ from now on, while the 331 model with a new left handed neutral fermion we call 331LHN. We present these two possibilities below and highlight their differences (There is also the Simple 331 model (Dong et al., 2014), but we are not considering it here either once it was shown to be ruled out by electroweak precision data (Dong and Si, 2014)).

\section{RH $v$ and 331LHN Content}

In the 331RHv model the leptons are arranged in triplet and in singlet representations of $\mathrm{SU}(3)_{\mathrm{L}}$ as follows:

$$
f_{a L}\left(\begin{array}{c}
v_{a L} \\
e_{a L} \\
\left(v_{a R}\right)^{C}
\end{array}\right) \sim(1,3-1 / 3), e_{a R} \sim(1,1-1)
$$

where, the index a labels the three known families, $a=1$, 2, 3 and the transformation rule under the gauge group is indicated in parentheses. Concerning the quark sector, we choose the first two families in an anti-triplet representation of $\mathrm{SU}(3)_{\mathrm{L}}$, while the third one transforms as a triplet with the following content:

$$
\begin{aligned}
& Q_{i L}\left(\begin{array}{c}
d_{i} \\
-u_{i} \\
d_{i}^{\prime}
\end{array}\right) \sim(3-\overline{3}, 0), u_{i R} \sim(3,1,2 / 3) \\
& d_{i R} \sim(3,1,-1 / 3), d_{i R}^{\prime} \sim(3,1,-1 / 3) \\
& Q_{3 L}\left(\begin{array}{c}
u_{3} \\
d_{3} \\
u_{3}^{\prime}
\end{array}\right) \sim(3,3,-1 / 3) u_{3 R} \sim(3,1,2 / 3) \\
& d_{3 R} \sim(3,1-1 / 3), u_{3 R}^{\prime} \sim(3,1,2 / 3)
\end{aligned}
$$

where, the index $\mathrm{i}=1,2$ refers to the first two generations. The primed fields are new heavy quarks, actually, leptoquarks since they also carry lepton number (This property is a trademark in 331 models due to the fact that these quarks interact with the new gauge bosons that carry lepton number).

In order to engender the electroweak symmetry breaking, generating the gauge boson and fermion masses, we need a minimum of three scalar triplets (A version with only two scalar triplets exists (Huong et al., 2011), but like those models in (Fregolente and Tonasse, 2003), it has not been presented a symmetry to stabilize the CDM candidate. Besides, its lifetime is smaller than necessary to be compatible with a good CDM candidate (Ackermann et al., 2012), unless a huge fine tuning is adopted to make it reasonable), whose field distribution is:

$X=\left(\begin{array}{c}X^{0} \\ X^{-} \\ X^{\prime 0}\end{array}\right),\left(\begin{array}{l}\eta^{0} \\ \eta^{-} \\ \eta^{\prime 0}\end{array}\right), \rho\left(\begin{array}{c}\rho^{+} \\ \rho^{0} \\ \rho^{\prime+}\end{array}\right)$

With $\eta$ and $X$ both transforming as $(1,3,-1 / 3)$ and $\rho$ transforming as $(1,3,2 / 3)$ under the 331 symmetry. These scalars are supposed to acquire VEV in the following fashion, in order to ignite the spontaneous symmetry breaking without promoting the lepton number spontaneous violation: 


$$
\eta^{0}, \rho^{0}, X^{\prime 0} \rightarrow \frac{1}{\sqrt{2}}\left(v_{\eta, \rho, X^{\prime}}+R_{\eta, \rho, X^{\prime}}+i I_{\eta, \rho, X^{\prime}}\right)
$$

The 331LHN model presents almost exactly the same content except for a small change. The leptonic content is instead:

$$
\begin{aligned}
& f_{a L}=\left(\begin{array}{c}
v_{a L} \\
e_{a L} \\
N_{a L}
\end{array}\right) \sim(1,3,-1 / 3) \\
& e_{a R} \sim(1,1-1), N_{a R} \sim(1,1,0)
\end{aligned}
$$

where, we have just changed the right handed neutrino, $\left(v_{a R}\right)^{C}$, by a left handed neutral fermion, $N_{a L}$, besides adding its singlet right handed counterpart, $\mathrm{N}_{\mathrm{aR}}$, to the spectrum. Nothing changes in the quark content. It may seem an insignificant change but we will see that there are implications for the CDM phenomenology.

A simplified Yukawa lagrangian and scalar potential is obtained in these models by imposing the following discrete symmetry:

$$
\left(X, \rho, e_{a R}, u_{a R} d_{i R}^{\prime}, Q_{3 L}\right) \rightarrow-\left(X, \rho, e_{a R}, u_{a R} d_{i R}^{\prime}, Q_{3 L}\right)
$$

The most general scalar potential can then be written as:

$$
\begin{aligned}
& V(\eta, \rho, X)=\mu_{X}^{2} X^{2}+\mu_{\eta}^{2} \eta^{2}+\mu_{\rho}^{2} \rho^{2}+\lambda_{1} X^{4}+\lambda_{2} \eta^{4}+\lambda_{3} \rho^{4}+ \\
& \lambda_{4}\left(X^{\dagger} X\right)\left(\eta^{\dagger} \eta\right)+\lambda_{5}\left(X^{\dagger} X\right)\left(\rho^{\dagger} \rho\right)+\lambda_{6}\left(\eta^{\dagger} \eta\right)\left(\rho^{\dagger} \rho\right)+ \\
& \lambda_{7}\left(X^{\dagger} \eta\right)\left(\eta^{\dagger} X\right)+\lambda_{8}\left(X^{\dagger} \rho\right)\left(\rho^{\dagger} X\right)+\lambda_{9}\left(\eta^{\dagger} \rho\right)(\rho \dagger \eta) \\
& -\frac{f}{\sqrt{2}} \epsilon^{i j k} \eta_{i} \rho_{j} X_{k}+\text { H.c }
\end{aligned}
$$

Which is the same for both models, while the Yukawa interactions for the 331RHv model writes:

$$
\begin{aligned}
& -L^{Y}=f_{i j} \bar{Q}_{i L X^{*}} d_{j R}^{\prime}+f_{33} \bar{Q}_{3 L X} X u_{3 R}^{\prime}+g_{i a} \bar{Q}_{i L} \eta^{*} d_{a R} \\
& +h_{3 a} \bar{Q}_{3 L} \eta u_{a R}+g_{3 a} \bar{Q}_{3 L} \rho d_{a R} \\
& +h_{i a} \bar{Q}_{i L} \rho * u_{a R}+G_{a a} \bar{f}_{a L} \rho e_{a R}+H . c .
\end{aligned}
$$

The 331LHN model, on the other hand, has one additional term to be included in the Yukawa lagrangian (A Majorana mass term for the right handed neutral fermion could be included as well, since it is sterile, but that is not relevant for the CDM analysis):

$$
-L^{Y} \supset+g_{a b}^{\prime} \bar{f}_{a L X} N_{b R}+\text { h.c. }
$$

where, the new neutral right-handed fermion fields, $N_{a R}$, are odd under the assumed $Z_{2}$ symmetry. This set of Yukawa interactions, after spontaneous symmetry breaking, allows for mass terms to all fermions, except the active neutrinos which, it may be assumed, obtain their masses through effective dimension-five operators according to (Dias et al., 2005).

Concerning the gauge sector, both models recover the usual SM gauge bosons, $\mathrm{W}^{ \pm}, Z^{0}, \gamma$ and contain five additional vector bosons, $\mathrm{V}^{ \pm}, \mathrm{U}^{0}, U^{0 \dagger}$ and $\mathrm{Z}$, (Montero et al., 1993), with masses around $1 \mathrm{TeV}$. The kinetic terms involving these gauge bosons imply new neutral and charged currents (not shown here) that, together with the Yukawa couplings in Equations (8) and (9), reveal an interesting feature of some of the new fields, they carry two units of lepton number (Depending on the nature of the third component of the leptonic triplet, this assignment can change to one unit when this component carries no lepton number (Dong et al., 2013; 2010; Kelso et al., 2014)):

$$
\begin{aligned}
& L\left(V^{+}, U^{\dagger 0}, u_{3}^{\prime}, \mu^{\prime 0}, \rho^{\prime+}\right)=-2, \\
& L\left(V^{-}, U^{0}, d_{i}^{\prime}, X^{0}, X^{-}\right)=+2
\end{aligned}
$$

This assignment is such that the lagrangian is lepton number conserving.

\section{Mass Spectrum}

With the lagrangian defined we can determine the mass spectrum of these models. As already discussed, all fermions acquire mass from the Yukawa terms in Equations (8) and (9) or higher dimension effective operators (the standard neutrinos). Let us focus here on the new neutral fermions of 331LHN model. In the absence of a Majorana mass term for the $\mathrm{N}_{\mathrm{L} / \mathrm{R}}$, Equation (9) gives only a Dirac mass for these fermions which is:

$$
m_{D}=\frac{g_{a b}^{\prime}}{\sqrt{2}} v_{x^{\prime}}
$$

At times, it may be appropriate to add a Majorana mass term for these fermions though, $\frac{M}{2} \bar{N}_{b R}^{c} N_{b R}$. This is the case of (Kelso et al., 2014), where the neutral fermion undergoes a seesaw mechanism and the heavier component plays the role of a mother particle that decays into a CDM particle during the radiation era to mimic relativistic species that may be showing up in several cosmological data (see for example (Ade et al., 2014) and references therein). In this case we would have two mass eigenstates:

$N_{L}^{\prime}=N_{L}+\frac{m_{D}}{M} N_{R}^{c}$ and $N_{R}^{\prime}=N_{R}-\frac{m_{D}}{M} N_{L}^{c}$

With respective eigenvalues:

$M_{N_{L}^{\prime}}=-\frac{m_{D}^{2}}{M}$ and $N_{R}^{\prime}=M_{N_{R}^{\prime}}=M$ 
By supposing that the Majorana mass emerges at very high energy scale, $M>>m_{D}, N_{L}$ becomes the light component and $\mathrm{N}_{\mathrm{R}}$ the heavy one.

Concerning the new quarks, according to Equation (8) their masses are proportional to the heavy VEV, $v_{X}$, and we assume they are heavier enough (few $\mathrm{TeV}$ ) so as to assure that one of the new neutral fields can be a stable CDM candidate in the model.

The extra gauge bosons have masses:

$$
\begin{aligned}
& m_{V}^{2}=m_{U 0}^{2}=\frac{1}{4} g^{2}\left(v_{X^{\prime}}^{2}+v^{2}\right), . \\
& m_{Z^{\prime}}^{2}=\frac{g^{2}}{4\left(3-4 s_{W}^{2}\right)}\left[4 c_{W}^{2} v_{X^{\prime}}^{2}+\frac{v^{2}}{c_{W}^{2}}+\frac{v^{2}\left(1-2 S_{W}^{2}\right)^{2}}{c_{W}^{2}}\right]
\end{aligned}
$$

where, $g$ is the standard electroweak gauge coupling, $\mathrm{s}_{\mathrm{W}}$ (and $c_{\mathrm{W}}$ ) refers to the Sine (and Cosine) of the electroweak mixing angle and $v=\frac{v_{\eta}}{\sqrt{2}}=\frac{v_{\rho}}{\sqrt{2}}$ is the EWSM breaking scale.

Given the approximations made over the parameters to obtain analytic expressions for the massive CP-even scalar eigenstates, described in (Pires and Silva, 2007; Mizukoshi et al., 2011), we have:

$$
H=\frac{\left(R_{\eta}+R_{\rho}\right)}{\sqrt{2}}, S_{1}=R_{X^{\prime}}, S_{2}=\frac{\left(R_{\eta}+R_{\rho}\right)}{\sqrt{2}}
$$

With the respective eigenvalues:

$$
\begin{aligned}
& M_{H}=\sqrt{3 \lambda_{2} u}, \\
& M_{S 1}=\sqrt{\frac{v^{2}}{4}+2 \lambda_{1} v_{X^{\prime}}^{2},} \\
& M_{S 2}=\sqrt{\frac{1}{2}\left(v_{X^{\prime}}^{2}+2 v^{2}\left(2 \lambda_{2}-\lambda_{6}\right)\right)}
\end{aligned}
$$

Here, $\mathrm{H}$ is identified with the recently detected Higgs boson (Aad et al., 2012), which is the lightest CP-even eigenstate. There is one massive CP-odd scalar field whose mass eigenstate is:

$$
P_{1} \approx \frac{v}{v_{X^{\prime}}} I_{X^{\prime}}+\frac{\left(I_{\eta}+I_{\rho}\right)}{\sqrt{2}}
$$

With the corresponding mass eigenvalue:

$$
M_{P 1}=\sqrt{\frac{1}{2}\left(v_{X^{\prime}}^{2}+\frac{v^{2}}{2}\right)}
$$

Besides the Goldstone bosons absorbed by the massive gauge fields and not shown here, we also have one last neutral complex scalar whose mass eigenstate is: $\phi \approx\left(\frac{v}{v_{x}^{\prime}} X^{* 0}+\eta^{\prime 0}\right)$

And its respective eigenvalue:

$m_{\phi}=\sqrt{\frac{\left(\lambda_{7}+\frac{1}{2}\right)}{2}\left[v^{2}+v_{x^{\prime}}^{2}\right]}$

Finally, the charged scalars are given by the following mass eigenstates:

$$
\begin{aligned}
& h_{1}^{-}=\frac{1}{\left(1+\frac{v^{2}}{v_{x^{\prime}}^{2}}\right)}\left(\frac{v}{v_{x^{\prime}}} X^{-}+\rho^{\prime-}\right) \\
& h_{2}^{-}=\frac{1}{\sqrt{2}}\left(\eta^{-}+\rho^{-}\right)
\end{aligned}
$$

Whose masses are, respectively:

$$
\begin{aligned}
& M_{h_{1}^{-}}^{2}=\frac{\lambda_{8}+\frac{1}{2}}{2}\left(v^{2}+v_{x^{\prime}}^{2}\right), \\
& M_{h_{2}^{-}}^{2}=\frac{v_{x^{\prime}}^{2}}{2}+\lambda_{g} v_{2}
\end{aligned}
$$

This completes the whole mass spectrum that is necessary to select the CDM particle in the 331 models here reviewed. Next we analyze the viable candidates considering peculiarities of each version of 331 models with neutral fermions in the leptonic triplets.

\section{CDM In 331 Models}

The particle physics explanation to the CDM problem demands a particle (or many) that possibly does not interact with most of the SM particles, if any. If this interaction happens to be similar in strength to the weak interaction and the candidate is stable (or meta-stable) with mass in the range of few $\mathrm{GeV}$ to some $\mathrm{TeV}$, it is called Weakly Interacting Massive Particle (WIMP). Besides fulfilling these requirements, such WIMP has to produce the correct abundance as inferred by the Cosmic Microwave Background radiation (CMB) power spectrum as measured by WMAP and Planck satellites (Ade et al., 2014; Bennett et al., 2003). The combined data of these experiments result in the CDM abundance, within $68 \%$ C.L.:

$0.1172<\Omega_{D M} h^{2}<0.1226$

Finally, current direct detection experiments have reported no convincing WIMP signature (Bernabei et al., 2008; Ahmed et al., 2010; Aalseth et al., 2011), putting 
bounds on the maximum WIMP-nucleon scattering cross section (Ahmed et al., 2010; Akerib et al., 2006; Aprile et al., 2011; Akerib et al., 2014). Thus, besides building a model that yields a WIMP that does not overpopulate the Universe, considering the upper bound in Equation (23), it is imperative that the parameters producing the appropriate thermal averaged WIMP annihilation cross section also comply with the upper bounds from detection experiments. Next we discuss the two models, 331RHv and 331LHN under the light of the WIMP paradigm.

\section{WIMP in $331 R H v$}

In the 331RHv model of (Pires and Silva, 2007), the particle that accomplishes these criteria is the excitation of the lightest bilepton field, which for an appropriate range of parameter space, turns out to be the scalar, $\phi$. This particular choice was made based on the fact that this scalar can only couple to another bilepton and if it is the lightest of its kind, lepton number conservation, together with the assumed $Z_{2}$ symmetry in Equation (6), would guarantee its stability. Notice that according to Equation (8), there is no WIMP-lepton coupling since lepton number is assumed to be conserved by the tree level lagrangian and the vacuum. The authors have shown that there is room in the parameter space for a WIMP that gives the right abundance and is in agreement with direct detection searches. However, the claimed $Z_{2}$ symmetry that precludes some terms in the scalar potential is spontaneously broken, since the neutral components of $\mathrm{X}$ and $\rho$ fields acquire VEV, which could jeopardize this scheme through unwanted operators leading to an unstable $\phi$. It happens that, even after the spontaneous breaking of $Z_{2}$, there is an apparent symmetry that operates on the bilepton scalars, since $\phi$ only couples to other bileptons, keeping the WIMP stable at tree level (Effective dimension five operators were supposed to generate neutrinos masses in the model (Dias et al., 2005; Pires and Silva, 2007), suppressed by high grand unification scale though, $\Lambda_{\mathrm{GUT}} \simeq 10^{14} \mathrm{GeV}$. One of them gives a Dirac mass term for the neutrinos $\frac{y^{\prime \prime}}{\Lambda_{G U T}}\left(f_{a L}^{\bar{c}} X^{*}\right)\left(\eta^{\dagger} f_{b L}\right) h . c$ and would imply the fast WIMP decay into two neutrinos with a lifetime roughly about few seconds. This operator explicitly breaks the $Z_{2}$ symmetry in the model though and does not represent any danger to WIMP stability). Actually, when lepton number is a global symmetry it may be broken by gravitational effects and dangerous operators suppressed by Planck scale, $\Lambda_{\mathrm{Pl}} \approx 10^{19} \mathrm{GeV}$, may arise to destroy the WIMP stability (Baek et al., 2013). Nevertheless, we cannot envisage any effective operator that would lead to a WIMP lifetime smaller than the allowed limit, $\tau \gtrsim 10^{26-30} s$ (Ackermann et al., 2012). By naively considering the most dangerous possible effective operator (The authors in (Chang and Long, 2006) wrote down the terms in the scalar potential that violate lepton number at tree level, which are not present in the potential Equation (7) due to (spontaneously broken) $\mathrm{Z}_{2}$ symmetry in Equation (6), but would reappear here when combined with another $\mathrm{Z}_{2}$ violating term):

$\frac{1}{\Lambda_{P l}^{2}} \in_{i j k} \overline{\left(f_{a L}\right) i}\left(f_{b L}^{C}\right)_{j} \rho_{k}^{*} X^{\dagger} \eta+h . c$.

The model was tested under the light of WMAP-3 year run results (Yao, 2006), concerning the relic CDM abundance and direct detection experiments, CDMS $(2004+2005)$ and XENON10 (Akerib et al., 2006), besides some projected experiments (Aprile et al., 2011; Schnee et al., 2005; Aprile et al., 2005). The analysis was rather crude, since several of the parameters of the model were fixed instead of using a scan (This drawback was to be fixed only later in a study of the $331 \mathrm{LHN}$ model (Cogollo et al., 2014). Also, the relic abundance was computed by using the approximate analytic Boltzmann equation (Jungman et al., 1996; Kolb and Turner, 1990). Nevertheless, the results have shown that there exists a restrict range of parameters guaranteeing that the scalar $\phi$ is the lightest bilepton and also a good WIMP candidate, furnishing the correct abundance and safe from direct detection, as presented in Fig. 1. Basically, a WIMP of $600 \mathrm{GeV}$ to $1 \mathrm{TeV}$ was probed in the 331RHv model and the concordance with relic abundance, as measured by WMAP, demanded a $\mathrm{SU}(3)_{\mathrm{L}} \otimes \mathrm{U}(1)_{\mathrm{N}}$ breaking scale, $v_{x^{\prime}} \gtrsim 1.3 \mathrm{TeV}$. Those results are still out of the sensitivity range of current direct detection experiments, as is the case of XENON100 (Aprile et al., 2011), but may be tested soon with augmented detector sensitivity (Aprile et al., 2005; LUX/LZ, 2012). We can infer that a WIMP mass higher than $1 \mathrm{TeV}$ may emerge from an update once bounds on the $Z^{\prime}$ mass imply a higher value $v_{x^{\prime}}>5.5 \mathrm{TeV}$ (Coutinho et al., 2010).

\section{WIMP in $331 L H N$}

Another variation with a second neutral fermion in the 331 leptonic triplet, that allows for a WIMP in the spectrum was considered in (Mizukoshi et al., 2011), the 331LHN model. As we mentioned, the difference between the 331RHv and the 331LHN is in the character of the third component of leptonic triplet, which in the later model is a new neutral left handed fermion, $\mathrm{N}_{\mathrm{L}}$, besides its right handed component, $\mathrm{N}_{\mathrm{R}}$, as a singlet under the gauge symmetry. A new symmetry was identified that transforms only the fields which are typically associated to the EWSM extended symmetry group. It was a global symmetry, $\mathrm{U}(1)_{\mathrm{G}}$ and the fields transforming non-trivially under it are given the following G-charge: 


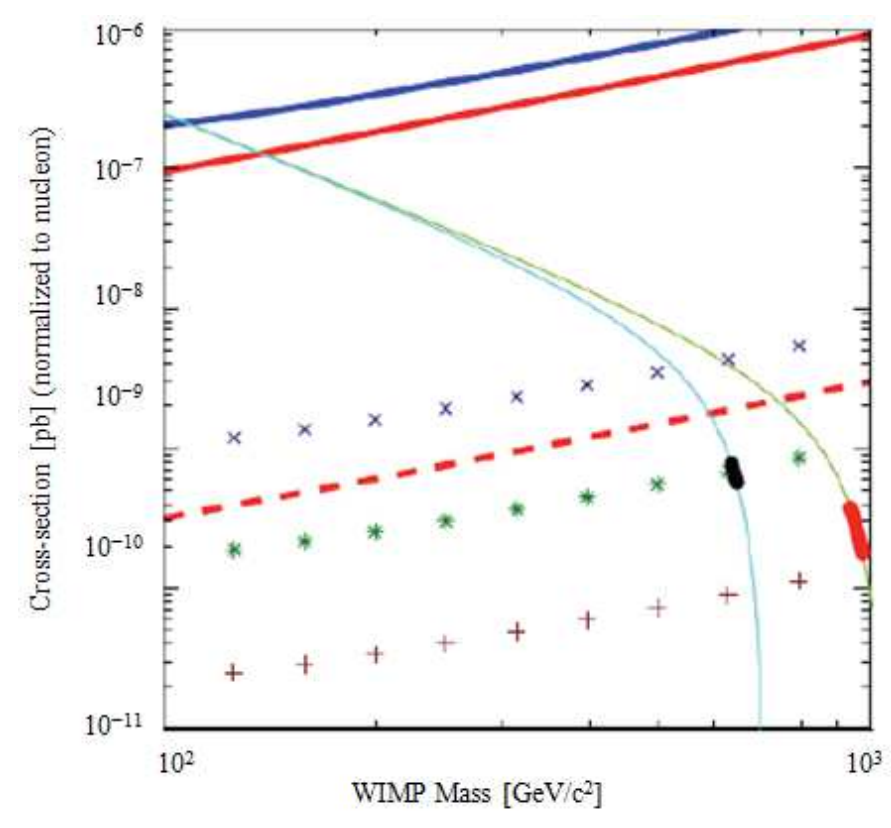

DATA listed top to bottom on plot

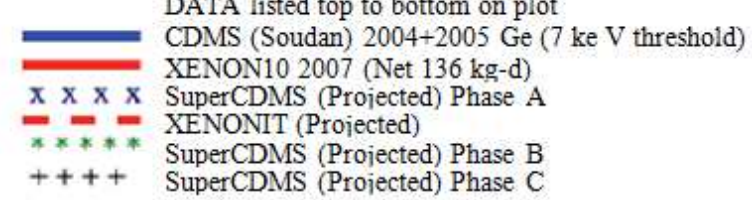

Fig. 1. Spin-independent WIMP-nucleon elastic scattering cross section sensitivity (current and projected) from CDMS and XENON Collaborations. The two thin lines crossing the data represent the SI WIMP-nucleon cross section of the 3-3-1RHv model as a function of the WIMP mass. The upper curve is for $v_{X^{\prime}}=3 \mathrm{TeV}$ and the lower one is for $v_{X^{\prime}}=2 \mathrm{TeV}$, both taken for $v_{q^{\prime}}=1.5$ $\mathrm{TeV}$. The thicker regions on these lines are those in agreement with the bounds on CDM imposed by WMAP 3-year run. Figure extracted from (Pires and Silva, 2007)

$G\left(\bar{N}_{L / R}, \bar{u}_{3 L / R}^{\prime}, d_{i L / R}^{\prime}, V_{\mu}^{-}, U_{\mu}^{-}, X^{0}, X^{-}, \eta^{\prime 0^{*}}, \rho^{\prime-}\right)=+1$

Notice that, except for $\mathrm{N}_{\mathrm{L} / \mathrm{R}}$, all fields that carry $\mathrm{G}-$ charge are bileptons and, again, in some sense it seems that lepton number is somehow involved in this new symmetry, without any clear identification of its role yet. The G-symmetry is claimed to stabilize the WIMP, which can be any electrically neutral G-charged field, $\mathrm{U}^{0}, \mathrm{~N}_{\mathrm{a}}$ and $\phi$ recalling that this last one is a combination of $\mathrm{X}^{0^{*}} \eta^{0}$, according to Equation (19). The non-hermitean gauge boson, $\mathrm{U}^{0}$, is not interesting from the CDM point of view, since it is extremely underabundant, so that it is chosen to be heavier than the lightest neutral fermion, labeled $\mathrm{N}_{1}$ and the scalar field, $\phi$. These cannot be coexisting CDM particles, then both possibilities were separately studied in (Mizukoshi et al., 2011). Here we face the same dilema as before in what concerns the symmetry to protect the WIMP from decay. Being a global symmetry, gravity effects can cast doubts about the WIMP stability, a point not considered in the original work. This is an important issue for the $\mathrm{N}_{1}$ since the following non-renormalizable operator emerges at planck scale (Baek et al., 2013) and promotes its decay into standard neutrinos:
$\frac{\lambda_{N 1 R}}{\Lambda_{P l}} \overline{N_{1 R}} D f_{1 L} \eta^{\dagger}+$ h.c.

Then, although the results concerning abundance and direct detection allow for a $\mathrm{N}_{1 \mathrm{R}}$ WIMP in the model, it is not realistic since the above operator would demand it to be too much lighter (less than few $\mathrm{keV}$ (Baek et al., 2013) than the TeV scale pointed in (Mizukoshi et al., 2011) in order to stay stable enough.

In this framework, there seems to remain only one interesting WIMP in this model, the scalar $\phi$. We have to look for the main dangerous effective operator that breaks $\mathrm{U}(1)_{\mathrm{G}}$ at Planck scale and still preserves the gauge symmetry. Such operators were mentioned in that work having a grand unification scale in mind, they would give Majorana mass for the neutrinos and were discarded once they explicitly violate $\mathrm{U}(1)_{\mathrm{G}}$ :

$\frac{y_{a b}^{\prime}}{\Lambda_{G U T}} \overline{f_{a L}^{c}} X^{*} X^{\dagger} f_{b L}+\frac{y_{a b}^{\prime \prime}}{\Lambda_{G U T}}\left(f_{a L}^{\bar{c}} X^{*}\right)\left(\eta^{\dagger} f_{a L}\right)+$ h.c.

However, they must be considered at Planck scale. Differently from the $331 \mathrm{RH} v$ model, where the $Z_{2}$ 
symmetry is supposed to be remnant from a gauge theory (not broken by gravity effects), in the 331LHN such an argument does not work for the global $\mathrm{U}(1)_{\mathrm{G}}$ and the second operator in Equation (27) would lead to fast decay of $\phi$ into neutrinos, unless the dimensionless coupling, $y$ " be unnaturally tiny, which would definitely turn this model awkward in what concerns the CDM problem.

That is not the final answer though. A smart solution exists for the choice of the correct symmetry that guarantees the existence of a WIMP in this model, which is based on the observation that the new neutral fermions and the bileptons carry the "wrong" lepton number (Chang and Long, 2006). Namely, the third component of the leptonic triplet carries a lepton number which is generally the opposite of its partners in the multiplet in the 331RHv model and it can be taken as null in the 331LHN (Dong et al., 2010), while the new quarks, some gauge bosons and scalar fields also carry lepton number in contrast to EWSM similar fields. This possibility lead to an enlargement of the 331 gauge group by an extra U(1) gauge symmetry, called 3311 model which, after spontaneous breakdown, still has a discrete $Z_{2}$ symmetry that remains unbroken and coincides with the R-parity symmetry of supersymmetry (Dong et al., 2013):

$W=(-1)^{3(B-L)+2 s}$

Called W-parity (W referring to wrong lepton number), where $\mathrm{B}$ is the baryon number, $\mathrm{L}$ is the lepton number and $\mathrm{s}$ is the spin of the field. Then, by assuming this unbroken $\mathrm{W}$-parity and considering the quantum numbers assigned to the $331 \mathrm{LHN}$ fields we get the odd transforming fields under this $\mathrm{Z}_{2}$ (Dong et al., 2013; Kelso et al., 2014; Profumo and Queiroz, 2014):

$W\left(N_{L / R}, d_{i}^{\prime}, u_{3}^{\prime}, \rho^{\prime \pm}, \eta^{\prime 0}, X^{0}, X^{ \pm}, V^{ \pm}, U^{0}\right)=-1$

Being an exact symmetry at low energies, it guarantees the stability of the lightest particle that transforms nontrivially under it, which means that either $\phi$ or $\mathrm{N}$ can be a (non-concomitant) realistic WIMP. Those two particles were extensively studied in this way and besides the simple analysis concerning their relic abundance and direct detection (Mizukoshi et al., 2011), several complementary interesting analyses were pursued that we summarize below.

When the complex scalar field, $\phi$, is chosen to be the lightestW-particle in the 331LHN model, it was shown to give the right abundance, scape direct detection and could also explain the gamma ray emission from the galactic center (Ruiz-Alvarez et al., 2012), as inferred by data from Fermi-LAT satellite (Hooper and Linden, 2011). This can be achieved for a WIMP mass between $25 \geq m_{\phi} \geq 40 \mathrm{GeV}$, when this WIMP predominantly annihilates into $b \bar{b}$ (more than $50 \%$ ), as reproduced in Fig. 2. Nevertheless, in the same work, this result was confronted with Higgs physics from LHC. Although such a light WIMP could solve the galactic center gamma ray emission in this model, it does not comply with Higgs decay as seen by LHC (Aad et al., 2012), since the model predicts a too high branching ratio into light WIMPs (whose mass is less than $60 \mathrm{GeV}$ ), close to $90 \%$, while predicting a too low branching ratio into two photons (Ruiz-Alvarez et al., 2012). Also, the scalar WIMP mass was further constrained in this model by considering bounds on the $Z^{\prime}$ mass, pushing the symmetry breaking scale of 331LHN to $v_{x^{\prime}} \gtrsim 10 \mathrm{TeV}$, which contrasted with recent LUX results on direct detection (Akerib et al., 2014), implies a lower bound to $\phi$ mass, $m_{\phi} \geq 500 \mathrm{GeV}$ (Cogollo et al., 2014), as can be seen in Fig. 3. Notice from that plot that XENON1T projected limit (Aprile et al., 2005) will be able to exclude the scalar dark matter mass range below 1 $\mathrm{TeV}$ in the 331LHN model.

Another interesting feature of this $331 \mathrm{LHN}$ model lies on the possibility to address the problem of extra radiation in the CMB spectrum, the so called Dark Radiation (see (Steigman et al., 1977) and references therein). A number of experiments have shown a mild but convincing preference for an effective neutrino number higher than the standard $\mathrm{N}_{\mathrm{eff}}=3.046$ (a nice compilation of results is presented in (Anchordoqui, 2014). The central value excess is around $\Delta N_{\text {eff }} \approx 0.5$ when considering the combined data from Planck + SPT + $\mathrm{WMAP}+\mathrm{ACT}+\mathrm{H}_{0}$ (Ade et al., 2014) and can be explained in the context of a heavy particle decaying into relativistic WIMPs at radiation dominated era (Ichikawa et al., 2007). This explanation fits well in the context of 331LHN model, since the lightest heavy neutral fermion, $\mathrm{N}_{1}$, can play the role of the mother particle, decaying into $\phi$ plus an active neutrino with parameters appropriate to produce only a fraction of relativistic WIMPs, remarking that this fraction must not exceed $0: 01 \%$ of the whole number of WIMPs so as to not jeopardize structure formation (Hooper et al., 2012). This approach was followed in (Kelso et al., 2014) and has been shown to be feasible for the $331 \mathrm{LHN}$ model while keeping consistency with the above mentioned constraints on $v_{x^{\prime}} \geq 10 \mathrm{TeV}$ and $m_{\phi} \geq 500 \mathrm{GeV}$ (Cogollo et al., 2014).

These results are evident from Fig. 4, where the mother particle is the lightest neutral fermion and the daughter particle is the scalar WIMP, which couple to each other with coupling strength $g_{11}^{\prime}$ originating from the Yukawa coupling in Equation (9). In this plot $f$ is the fraction of WIMP that is produced relativistically from $\mathrm{N}_{1}$ decay and $\Delta$ is a suppression factor that can account for a possible high relic number density of the mother particle, which may be the case if $g_{11}^{\prime}$ is to small, possibly pushing the model into the region where $\phi$ ceases to be stable (the red shaded area in the plot). 


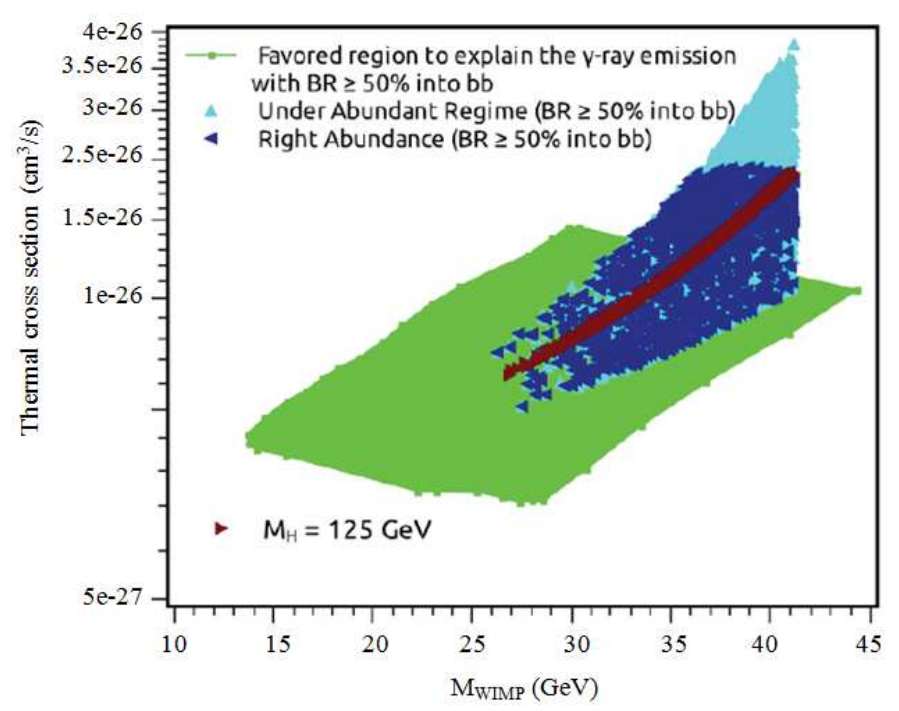

Fig. 2. Annihilation cross section as function of $M_{\phi}$ for $B R(b \bar{b}) \geq 50 \%$. The green region represents the favored region by the gamma-ray emission detected by Fermi-LAT in the galactic center (Hooper and Linden, 2011). Dark (light) blue points refer to the case where the WIMP provides the correct abundance (under abundant). All (dark+light) blue points are for $110 \mathrm{GeV}$ $\leq \mathrm{MH} \leq 150 \mathrm{GeV}$, while the brown points are for $\mathrm{MH}=125 \mathrm{GeV}$. Correct abundance means $0.098 \leq \Omega \mathrm{h}^{2} \leq 0.122$ while the under abundant regime is for $(0: 01 \leq \Omega \mathrm{h} 2 \leq 0.098)$. Figure taken from (Ruiz-Alvarez et al., 2012

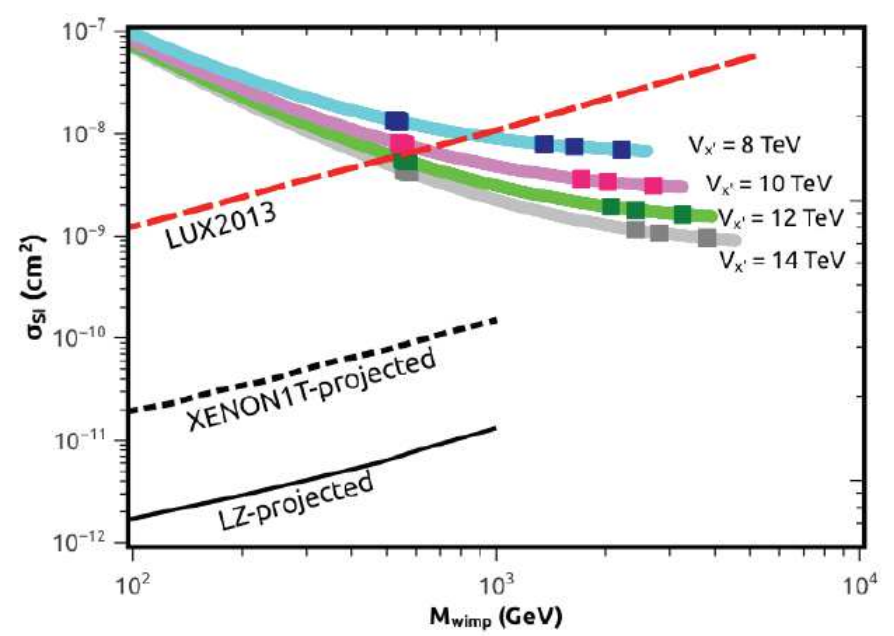

Fig. 3. WIMP-nucleon spin independent scattering cross section for $v_{X}=8,10,12,14 \mathrm{TeV}$. The dark points delimit the parameter space that yields the right abundance in accordance with Planck (Ade et al., 2014), Equation (23). The dashed red (black) curve is the LUX2013 (Akerib et al., 2014) (XENON1T-projected (Aprile et al., 2005) limit, while the solid black line is for the projected LZ limit (LUX/LZ, 2012). Figure extracted from (Cogollo et al., 2014)

The other interesting possibility is when one of the new neutral fermions, $\mathrm{N}_{\mathrm{a}}$, is the lightest odd $\mathrm{W}$-parity, becoming the CDM candidate. In this case, although there are regions in the parameter space compatible with the observed relic abundance for $m_{N} \approx 200 \mathrm{GeV}$, the WIMP is pushed into masses around $1 \mathrm{TeV}$ when confronted with direct detection experiments, implying a $331 \mathrm{LHN}$ symmetry breaking scale, $v_{x^{\prime}} \geq 4$ TeV (Mizukoshi et al., 2011), as can be seen from
Fig. 5. An interesting study considering the role of $Z$ ' in the WIMP-nucleon scattering amplitude, translated into bounds in the cross section coming from direct detection experiments, mainly the recent LUX results (Akerib et al., 2014) which, together with constraints from CDM relic abundance, forced $M_{Z} \gtrsim 2 \mathrm{TeV}$ for a WIMP mass around $1 \mathrm{TeV}$ (Profumo and Queiroz, 2014), as shown in Fig. 6. This further pushes the lower bound on the breaking scale, $v_{x^{\prime}} \geq \mathrm{TeV}$. 


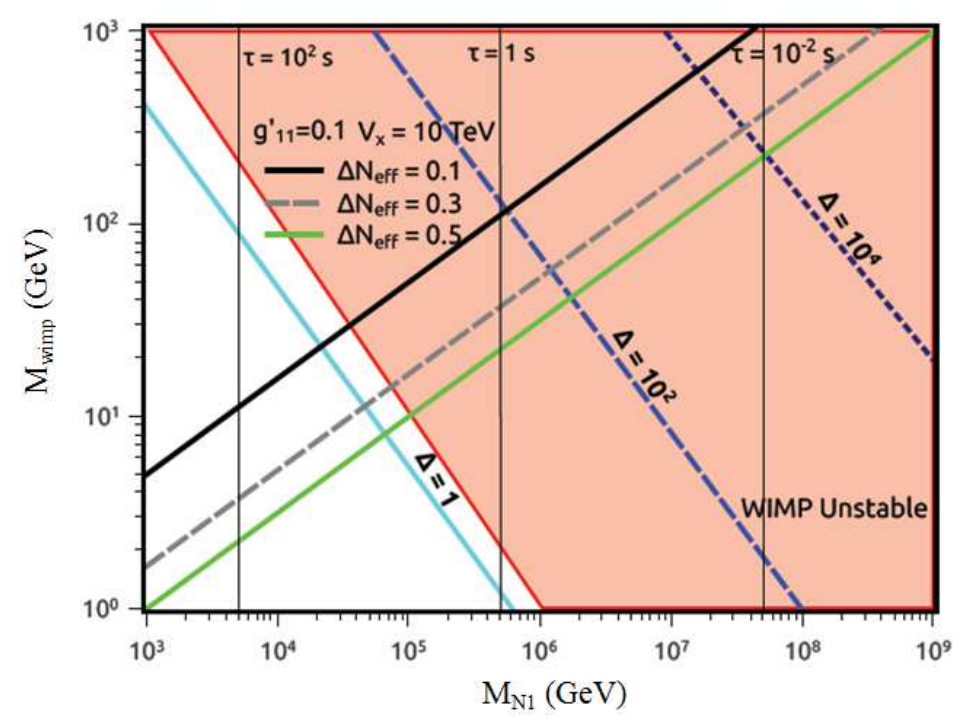

Fig. 4. The "mother-daughter" particle mass parameter space, for $g_{11}^{\prime}=1$. The vertical lines indicate constant values of the mother particle lifetime. The diagonal lines indicate the induced variation in the number of effective relativistic degrees of freedom $\Delta \mathrm{N}_{\mathrm{eff}}$ and the entropy dilution factor $\Delta$ needed to suppress the mother particle relic density. The cyan $\Delta=1$ line corresponds to standard cosmology without any entropy dilution needed. Here $v_{X}^{\prime}=10 \mathrm{TeV}$ and the red shaded region induces the WIMP decay. Figure extracted from (Kelso et al., 2014)

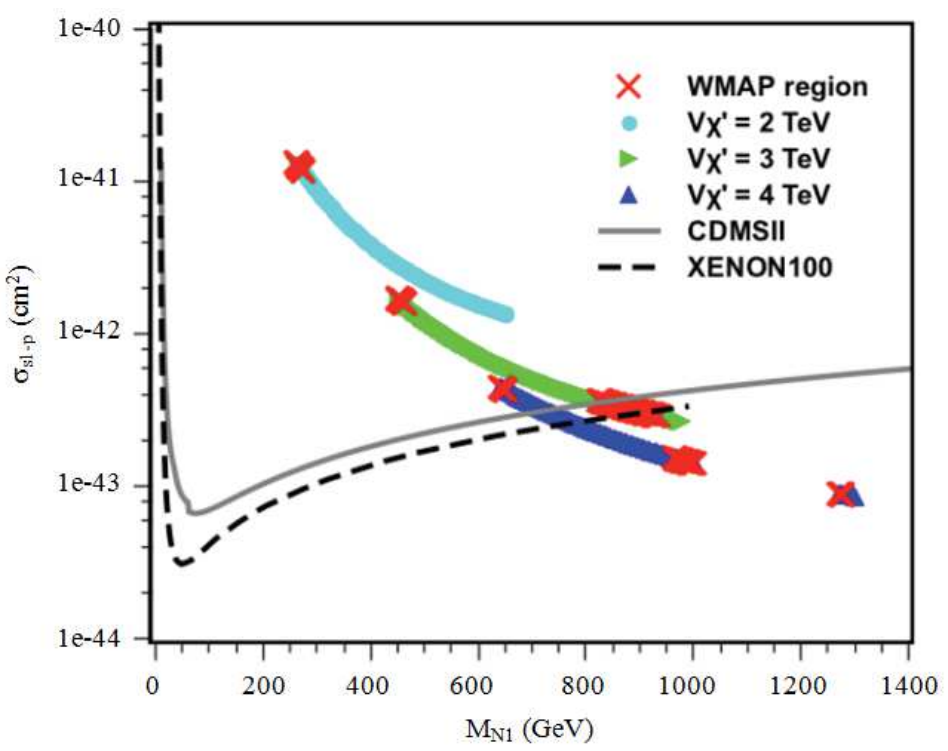

Fig. 5. The WIMP-proton cross section for N1. From top to bottom, the curves represent the variation of $v_{X}^{\prime}$ in the range $2 \mathrm{TeV} \leq v_{X}^{\prime} \leq 4$ $\mathrm{TeV}$. The data used in the exclusion curves were obtained using. Figure extracted from (Mizukoshi et al., 2011)

Observe that these constraints are an important outcome concerning this $331 \mathrm{LHN}$ model, since the bounds on MZ' obtained in (Coutinho et al., 2010) do not apply in the case $m_{N} \leq M_{Z}^{\prime} / 2$, where the main $Z^{\prime}$ decay channel into charged leptons is suppressed due to this preferred invisible channel into two WIMPs. It is another complementary connection from dark matter that should be taken into account in the specific case where the heavy neutral fermion is the CDM candidate in 331LHN (Profumo and Queiroz, 2014). Similar results, for both CDM candidates, were obtained in a phenomenological study of the 331LHN model with an extra gauge $U(1)$ group broken to the W-parity symmetry (Dong et al., 2014) (The WIMP candidates are exactly the same since this model is a simple enlargement of the gauge group by an abelian factor). 


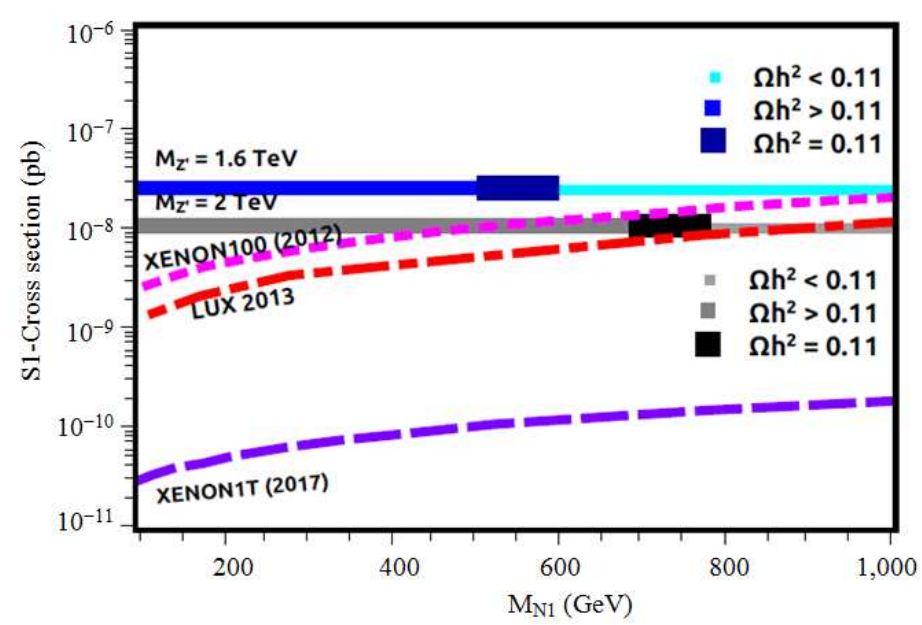

Fig. 6. $\mathrm{N}_{1}$ spin independent scattering cross section off nuclei. Figure extracted from (Profumo and Queiroz, 2014)

\section{Conclusion}

We have revisited the electroweak gauge extensions of the SM, the 331 models with neutral fermions, considering their ability to provide a good CDM candidate, more specifically, a WIMP. Two such models were considered, the 331RHv (Pires and Silva, 2007) and the 331LHN (Mizukoshi et al., 2011), differing by the fact that in the first model the neutral fermion is the right handed partner of the left handed neutrino, while in the second one it is a new kind of neutral fermion that may not carry lepton charge. Both models possess a complex scalar CDM candidate, while the 331LHN offers an additional nonconcomitant candidate, the new neutral fermion. The properties that guarantee the existence of a WIMP were analyzed and essentially the main problem in these models has been finding the appropriate symmetry to protect the WIMP from decaying.

Such stability, as discussed, is not guaranteed for the $331 \mathrm{RNv}$, since the proposed symmetry is spontaneously broken. While lepton number seems to play some role in keeping the WIMP, $\phi$ in this model, stable at tree level, higher dimensional effective operators imply the WIMP decay due to gravitational effects, with a lifetime $\tau \approx 10^{43} \mathrm{~s}$, big enough to consider it stable. Constraints from $Z$ ' mass push the symmetry breaking scale to $v_{x^{\prime}} \gtrsim 5.5 \mathrm{GeV}$ and, according to the results shown in (Pires and Silva, 2007), a scalar WIMP of mass around $1 \mathrm{TeV}$ would be compatible with relic CDM abundance and direct detection.

Considering the $331 \mathrm{LHN}$ model, the global $\mathrm{U}(1)_{\mathrm{G}}$ symmetry proposed in (Mizukoshi et al., 2011) was not suitable to provide the WIMP stability, once the same effective operators that respect the symmetries of the model and are employed to generate Majorana mass for the neutral fermions, would be induced by gravitational effects in what concerns lepton number violation. Differently from $331 \mathrm{RH} v$ model, such operators lead to fast WIMP decay, unless some huge fine tuning is claimed. However, a new discrete symmetry called W-parity, similar to R-parity in supersymmetric models, was implemented in this model when the neutral fermion in the leptonic triplet does not carry lepton number (Dong et al., 2013). It was first used as a remnant of a specific gauge symmetry in (Dong et al., 2013; Dong et al., 2014) and employed afterwards without worrying which gauge symmetry may be behind its survival at low energies (Kelso et al., 2014; Cogollo et al., 2014; Profumo and Queiroz, 2014). In this case, all results obtained before remain valid for the 331LHN model (Mizukoshi et al., 2011; Ruiz-Alvarez et al., 2012), since no relevant changes have affected the WIMP identity and properties.

The interesting outcomes of 331LHN CDM model reveal that the scalar WIMP, $\phi$, which could have a large mass range (Mizukoshi et al., 2011), could offer an explanation for the excess of gamma ray emission from the galactic center when $25 \leqslant m_{\phi} \leq 40 \mathrm{GeV}$. That was shown to be unrealistic when confronted with complementary phenomenology from recently observed Higgs physics (Ruiz-Alvarez et al., 2012), since the Higgs decay was predicted to be more than $90 \%$ into two WIMPs if $m_{\phi} \lesssim 60 \mathrm{GeV}$, completely ruled out by LHC data (Aad et al., 2012). Further analysis has shown that $m_{\phi} \geq 500 \mathrm{GeV}$ and $v_{x^{\prime}} \geq 10 \mathrm{TeV}$ when the bounds over the mass of the new neutral gauge boson, $Z^{\prime}$, were considered (Cogollo et al., 2014) in conjunction with recent constraints of $\mathrm{CDM}$ direct detection experiment LUX (Akerib et al., 2014). Besides, the model accommodates the right content to address a solution to the dark radiation problem through the out-ofequilibrium production of a fraction of the WIMP as relativistic species (Kelso et al., 2014).

Finally, when the WIMP from 331LHN model was chosen to be the lightest heavy neutral fermion, $\mathrm{N}_{1}$, the 
first analysis made already evident that it should have a mass around $1 \mathrm{TeV}$ to be consistent with relic abundance and direct detection experiments (Mizukoshi et al., 2011). The bounds on the symmetry breaking scale are weaker in this case, though. Since existent limits from LHC physics on the $Z^{\prime}$ mass (Coutinho et al., 2010) may not apply to this model for $M_{N} \lesssim 1 \mathrm{TeV}$, in which case $Z^{\prime}$ would prefer to decay invisibly into two WIMPs, a bound over the $Z^{\prime}$ mass was found in this specific case by considering LUX results, obtaining $M_{Z^{\prime}} \gtrsim 2 \mathrm{TeV}$ and thus, $v_{x^{\prime}} \gtrsim 5 \mathrm{TeV}$ (Profumo and Queiroz, 2014). It is worth to be mentioned that this result truly complements previous results on $Z^{\prime}$ mass (Coutinho et al., 2010) and shows the importance of $\mathrm{CDM}$ approach to electroweak precision phenomenology.

\section{Acknowledgment}

The author feels deeply grateful to his collaborators, Alex Dias, Carlos Pires and Farinaldo Queiroz. This work was supported by the Conselho Nacional de Desenvolvimento Cient'ifico e Tecnol'ogico (CNPq).

\section{Ethics}

This article is original and contains unpublished material. The corresponding author confirms that all of the other authors have read and approved the manuscript and no ethical issues involved.

\section{References}

Aad, G., T. Abajyan, B. Abbott, J. Abdallah and S.A. Khalek et al., 2012. Observation of a new particle in the search for the Standard Model Higgs boson with the ATLAS detector at the LHC. Phys. Lett., B, 716: 1-29. DOI: 10.1016/j.physletb.2012.08.020

Aalseth, C.E., P.S. Barbeau, N.S. Bowden, B. CabreraPalmer and J. Colaresi et al., 2011. Results from a search for light-mass dark matter with a p-type point contact germanium detector. Phys. Rev. Lett., 106: 131301-131301. DOI: 10.1103/PhysRevLett.106.131301

Ackermann, M., M. Ajello, A. Albert, L. Baldini and G. Barbiellini et al., 2012. Fermi LAT search for dark matter in gamma-ray lines and the inclusive photon spectrum. Phys. Rev. D, 86: 022002-022002.

Ade, P.A.R., R.W. Aikin, D. Barkats, S.J. Benton and C.A. Bischoff et al., 2014. BICEP2 I: Detection of b-mode polarization at degree angular scales. Phys. Rev. Lett., 112: 241101-241101. DOI: 10.1103/PhysRevLett.112.241101

Ahmed, Z., D.S. Akerib, S. Arrenberg, C.N. Bailey and D. Balakishiyeva et al., 2010. Dark matter search results from the CDMS II experiment. Science, 327: 1619- 1621. DOI: 10.1126/science. 1186112
Akerib, D.S., M.J. Attisha, C.N. Bailey, L. Baudis and D.A. Bauer et al., 2006. Limits on spin-independent interactions of weakly interacting massive particles with nucleons from the two-tower run of the cryogenic dark matter search. Phys. Rev. Lett., 96: 011302-011302. PMID: 16486434

Akerib, D.S., H.M. Araujo, X. Bai, A.J. Bailey and J. Balajthy et al., 2014. First results from the LUX dark matter experiment at the sanford underground research facility. Phys. Rev. Lett., 112: 091303091303. DOI: 10.1103/PhysRevLett.112.091303

Anchordoqui, L.A., 2014. Constraints on cosmological parameters from Planck and BICEP2 data. Cosmol. Nongalactic Astrophysics.

Aprile, E., K. Arisaka, F. Arneodo, A. Askin and L. Baudis et al., 2011. Dark matter results from 100 live days of XENON100 data. Phys. Rev. Lett., 107: 131302-131302. DOI: 10.1103/PhysRevLett.107.131302

Aprile, E., K.L. Giboni, P. Majewski, K. Ni and M. Yamashita et al., 2005. The XENON dark matter search experiment. New Astron. Rev., 49: 289-295. DOI: 10.1016/j.newar.2005.01.035

Baek, S., P. Ko and W.I. Park, 2013. Singlet portal extensions of the standard seesaw models to a dark sector with local dark symmetry. J. High Energy Phys. DOI: 10.1007/JHEP07(2013)013

Bennett, C.L., M. Halpern, G. Hinshaw, N. Jarosik and A. Kogut et al., 2003. First-Year Wilkinson Microwave Anisotropy Probe (WMAP)* Observations: Preliminary Maps and Basic Results. Astrophys. J. Suppl., 148: 1-1. DOI: 10.1086/377253

Bernabei, R., P. Belli, F. Cappella, R. Cerulli and C.J. Dai et al., 2008. First results from DAMA/LIBRA and the combined results with DAMA/NaI. Eur. Phys. J. C, 56: 333-333. DOI: 10.1140/epjc/s10052-008-0662-y

Caetano, W., C.A. de S. Pires, P.S. Rodrigues da Silva, D. Cogollo and F.S. Queiroz, 2013. Explaining ATLAS and CMS results within the reduced minimal 3-3-1 model. Eur. Phys. J. C, 73: 2607-2607. DOI: $10.1140 /$ epjc/s10052-013-2607-3

Chang, D. and H.N. Long, 2006. Interesting radiative patterns of neutrino mass in an $\mathrm{SU}(3)_{\mathrm{C}} \otimes \mathrm{SU}(3)_{\mathrm{L}} \otimes \mathrm{U}(1)_{\mathrm{X}}$ model with right-handed neutrinos. Phys. Rev. D, 73: 053006-053006. DOI: 10.1103/PhysRevD.73.053006

Cheng, H.S., J.L. Feng and K.T. Matchev, 2002. Kaluza-klein dark matter. Phys. Rev. Lett., 89: 211301-211301. DOI: 10.1103/PhysRevLett.89.211301

Cogollo, D., A.X. Gonzalez-Morales, F.S. Queiroz and P.R. Teles, 2014. Excluding the light dark matter window of a 331 model using LHC and direct dark matter detection data. JCAP, 1411: 002-002. 
Coutinho, Y.A., V.S. Guimaraes and A.A. Nepomuceno, 2010. Bounds on $Z^{\prime}$ from 3-3-1 model at the LHC energies. Phys. Rev. D, 87: 115014-115014.

Dias, A.G., C.A. de S. Pires and P.S. Rodrigues da Silva, 2003. Discrete symmetries, invisible axion and lepton number symmetry in an economic 3-3-1 model. Phys. Rev. D, 68: 115009-115009.

DOI: 10.1103/PhysRevD.68.115009

Dias, A.G., C.A.D.S. Pires and P.S. Rodrigues da Silva, 2005. Naturally light right-handed neutrinos in a 33-1 model. Phys. Lett. B, 628: 85-85. DOI: 10.1016/j.physletb.2005.09.028

Dodelson, S. And L.M. Widrow, 1994. Sterile neutrinos as dark matter. Phys. Rev. Lett., 72: 17-17.

Dong, P.V. and D.T. Si, 2014. Discriminating the minimal 3-3-1 models. Phys. Rev. D, 90: 117703-117703. DOI: 10.1103/PhysRevD.90.117703

Dong, P.V., D.T. Huong, F.S. Queiroz and N.T. Thuy, 2014. Phenomenology of the 3-3-1-1 model. Phys. Rev. D, 90: 075021-075021. DOI: 10.1103/PhysRevD.90.075021

Dong, P.V., H.T. Hung and T.D. Tham, 2013. 3-3-1-1 model for dark matter. Phys. Rev. D. DOI: 10.1103/PhysRevD.87.115003

Dong, P.V., L.T. Hue, H.N. Long and D.V. Soa, 2010. The 3-3-1 model with $\mathrm{A}_{4}$ flavor symmetry. Phys. Rev. D, 81: 053004-053004. DOI: 10.1103/PhysRevD.81.053004

Dong, P.V., N.T.K. Ngan and D.V. Soa, 2014. Simple 33-1 model and implication for dark matter. Phys. Rev. D, 90: 075019-075019. DOI: 10.1103/PhysRevD.90.075019

Dong, P.V., T.P. Nguyen and D.V. Soa, 2013. 3-3-1 model with inert scalar triplet. Phys. Rev. D, 88: 095014095014. DOI: 10.1103/PhysRevD.88.095014

Fregolente, D. and M.D. Tonasse, 2003. Self-interacting dark matter from a $\mathrm{SU}(3)_{\mathrm{L}} \otimes \mathrm{U}(1)_{\mathrm{N}}$ electroweak model. Phys. Lett., B, 555: 7-12. DOI: $10.1016 / \mathrm{S} 0370-2693(03) 00037-6$

Hooper, D. and T. Linden, 2011. Origin of the gamma rays from the galactic center. Phys. Rev. D. DOI: 10.1103/PhysRevD.84.123005

Hooper, D., F.S. Queiroz and N.Y. Gnedin, 2012. Nonthermal dark matter mimicking an additional neutrino species in the early universe. Phys. Rev. D, 85: 063513-063513. DOI: 10.1103/PhysRevD.85.063513

Huong, D.T., C.S. Kim, H.N. Long and N.T. Thuy, 2011. Probing dark matter in the economical 3-3-1 model. Cornell University Library.

Ichikawa, K., M. Kawasaki, K. Nakayama, M. Senami and F. Takahashi, 2007. Increasing the effective number of neutrinos with decaying particles. JCAP. DOI: $10.1088 / 1475-7516 / 2007 / 05 / 008$
Jungman, G., M. Kamionkowski and K. Griest, 1996. Supersymmetric dark matter. Phys. Rept., 267: 195-373. DOI: 10.1016/0370-1573(95)00058-5

Kelso, C., C.A.D.S. Pires, S. Profumo, F.S. Queiroz and P.S. Rodrigues da Silva, 2014. A 331 WIMPy dark radiation model. Eur. Phys. J. C, 74: 2797-2797. DOI: $10.1140 /$ epjc/s10052-014-2797-3

Kolb, E.W. and M.S. Turner, 1990. The early universe"front. Phys. 69: 1-547.

Ky, N.A., H.N. Long and D.V. Soa, 2000. Anomalous magnetic moment of muon in 3-3-1 models. Phys. Lett. B, 486: 140-146. DOI: 10.1016/S0370-2693(00)00696-1

LUX/LZ, 2012. LUX/LZ collaboration.

Martin, A., 2006. Dark matter in the simplest little higgs model. Cornell University Library.

Mizukoshi, J.K., C.A.D.S. Pires, F.S. Queiroz and P.S. Rodrigues da Silva, 2011. WIMPs in a 3-3-1 model with heavy sterile neutrinos. Phys. Rev. D, 83: 065024-065024.

DOI: 10.1103/PhysRevD.83.065024

Montero, J.C., F. Pisano and V. Pleitez, 1993. Neutral currents and Glashow-Iliopoulos-Maiani mechanism in $\mathrm{SU}(3)_{\mathrm{L}} \otimes \mathrm{U}(1)_{\mathrm{N}}$ models for electroweak interactions. Phys. Rev. D, 47: 2918-2918. DOI: 10.1103/PhysRevD.47.2918

Murayama, H., 2007. Physics beyond the standard model and dark matter. Cornell University Library.

Pires, C. A.D.S., F. Queiroz and P.S. Rodrigues da Silva, 2010b. Minimal SU(3) $)_{\mathrm{C}} \times \mathrm{SU}(3)_{\mathrm{L}} \times \mathrm{U}(1)_{\mathrm{N}}$ model with natural sub-eV neutrinos. Phys. Rev. D, 82: 065018065018. DOI: 10.1103/PhysRevD.82.065018

Pires, C.A.D.S. and O.P. Ravinez, 1998. Electric charge quantization in a chiral bilepton gauge model. Phys. Rev. D, 58: 035008-035008.

DOI: 10.1103/PhysRevD.58.035008

Pires, C.A.D.S. and P.S.R.D Silva, 2007. Scalar bilepton dark matter. JCAP, 0712: 012-012. DOI: $10.1088 / 1475-7516 / 2007 / 12 / 012$

Pires, D.S.C.A., F.S. Queiroz and P.S. Rodrigues da Silva, 2010a. Singlet Majorana fermion dark matter, DAMA, CoGeNT and CDMS-II. Phys. Rev. D, 82: 105014-105014. DOI: $10.1103 /$ PhysRevD.82.105014

Pisano, F. and V. Pleitez, 1992. SU(3)®U(1) model for electroweak interactions. Phys. Rev. D, 46: 410-410. DOI: 10.1103/PhysRevD.46.410

Profumo, S. and F.S. Queiroz, 2014. Constraining the $Z^{\prime}$ mass in 331 models using direct dark matter detection. Eur. Phys. J. C, 74: 2960-2960.

Ruiz-Alvarez, J.D., C.A.D.S. Pires, F.S. Queiroz, D. Restrepo and P.S. Rodrigues da Silva, 2012. Connection of gamma rays, dark matter and Higgs boson searches at the LHC. Phys. Rev. D, 86: 075011075011. DOI: 10.1103/PhysRevD.86.075011 
Schnee, R.W., D.S. Akerib, M.J. Attisha, C.N. Bailey and L. Baudis et al., 2005. The SuperCDMS Experimen. Cornell University Library.

Singer, M., J.W.F. Valle and J. Schechter, 1980. Canonical neutral-current predictions from the weak-electromagnetic gauge group $\mathrm{SU}(3) \times \mathrm{U}(1)$. Phys. Rev. D, 22: 738-738.

DOI: 10.1103/PhysRevD.22.738
Steigman, G., D.N. Schramm and J.E. Gunn, 1977. Phys. Lett. B, 66: 202-202.

Valle, J.W.F. and M. Singer, 1983. Lepton-number violation with quasi-Dirac neutrinos. Phys. Rev. D, 28: 540-540. DOI: 10.1103/PhysRevD.28.540

Yao, W.M., 2006. Review of particle physics. J. Phys. G, 33: 1-1232. DOI: 10.1088/0954-3899/33/1/001 